Document downloaded from:

http://hdl.handle.net/10251/54590

This paper must be cited as:

Marton Lluch, I.; Sánchez Galdón, Al.; Martorell Alsina, SS. (2015). Ageing PSA incorporating effectiveness of maintenance and testing. Reliability Engineering and System Safety. 139:131-140. doi:10.1016/j.ress.2015.03.022.

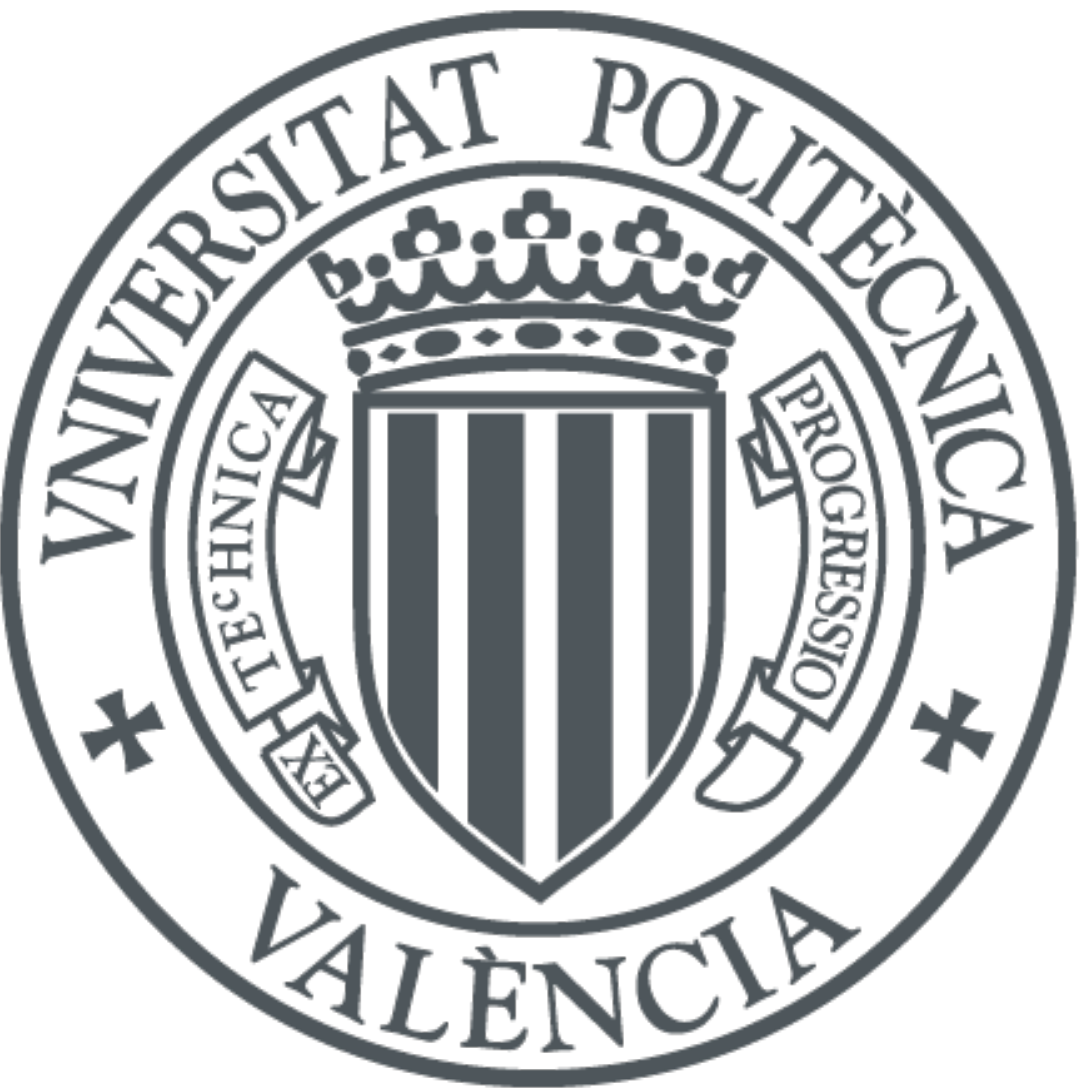

The final publication is available at

http://dx.doi.org/10.1016/j.ress.2015.03.022

Copyright Elsevier

Additional Information 


\title{
Ageing PSA incorporating effectiveness of maintenance and testing
}

\author{
I. Martón ${ }^{1}$, A.I. Sánchez², S. Martorell ${ }^{1, *}$ \\ ${ }^{1}$ Department of Chemical and Nuclear Engineering. Universitat Politècnica de València, Valencia, Spain \\ ${ }^{2}$ Department of Statistics and Operational Research. Universitat Politècnica de València, Valencia, Spain
}

${ }^{*}$ Corresponding author: S. Martorell (smartore@iqn.upv.es)

\begin{abstract}
This paper proposes a new approach to Ageing Probabilistic Safety Assessment (APSA) modelling, which is intended to be used to support risk-informed decisions on the effectiveness of maintenance management programs and technical specification requirements of critical equipment of Nuclear Power Plants (NPP) within the framework of the Risk Informed Decision Making according to R.G. 1.174 principles. This approach focuses on the incorporation of not only equipment ageing but also effectiveness of maintenance and efficiency of surveillance testing explicitly into APSA models and data. An example of application is presented, which centers on a critical safety-related equipment of a NPP in order to evaluate the risk impact of considering different approaches to APSA and the combined effect of equipment ageing and maintenance and testing alternatives along NPP design life. The risk impact of the several alternatives is quantified and the results shows that such risk depends largely on the model parameters, such as ageing factor, maintenance effectiveness, test efficiency.
\end{abstract}

Keywords: Risk, decision-making, ageing, PSA, asset management, design life management, life extension, long-term operation, maintenance, surveillance requirements, NPP equipment, periodic safety review. 


\section{INTRODUCTION}

One of the main challenges for 2020 horizon is to maintain fission technologies competitiveness by research and technological development necessary in order to allow a safe Long Term Operation (LTO) of the nuclear reactors in operation nowadays (GEN II and III) [1-3]. For example, in 2020 most of the Nuclear Power Plants (NPP) in Spain, which are Light Water Reactor (LWR) type, would be operating for around 40 years, which is the plant design life, and they will face the Periodic Safety Review (PSR) to obtain, eventually, life extension approval for the LTO from the regulatory body (usually for 10 more years).

During the design life, licensee of NPP must perform the necessary activities for ageing management of Structures, Systems and Components (SSC), which include consideration of measures for monitoring, control and mitigation of the mechanisms of physical ageing of SSC adopting the necessary maintenance in scope of asset management programs and surveillance testing activities within NPP technical specifications.

An important part of PSR involves the evaluation of risk impact of how current life management of ageing equipment, e.g. maintenance and testing programs, is successful in achieving the objective of allowing a safe operation along NPP design life. In particular, PSR close to the end of the NPP design life should involve the evaluation of at least, but not only, how ageing management along NPP design life would jeopardize NPP safety for the extended period. In addition, if necessary, it should be also evaluated the effectiveness of adopting an alternative ageing management plan, e.g. maintenance policy, or even, studying the risk impact of changing technical specification requirements, such as surveillance requirements, for the extended life in order to achieve the safety standards required to get approval from regulatory bodies for NPP long term operation.

\section{PROBABILISTIC SAFETY ASSESSMENT AND ITS APPLICATION}

Probabilistic Safety Analysis (PSA) is an essential tool for assessing, maintaining, assuring and improving the NPP safety. Nowadays, PSA is an efficient tool for evaluation of risk impact of changes to licensing basis [4] and in particular to surveillance requirements of NPP technical specifications within the framework of the Risk Informed Decision Making (RIDM) according to R.G. 1.174 principles [5, 6]. 
In a similar way, PSA can become also a relevant tool for the evaluation of the effectiveness of asset management programs from a risk viewpoint, e.g. searching for risk-efficient maintenance and testing programs of ageing equipment along NPP life (both design life and extended life). However, standard PSA does not address adequately important ageing and maintenance and testing issues necessary for it to play such a relevant role. For example, current practice in PSA modelling considers constant failure rates instead of explicit agedependent failure rates of safety related equipment. In addition, they do not formulate explicitly how a large variety of surveillance, maintenance and inspection programs, which are intended to mitigate or at least keep under control the effects of equipment ageing, impact on failure rates. The extent to which components are susceptible to ageing differs considerably depending on the particular life management program (maintenance and testing) and its effectiveness along the operational life of the NPP.

Therefore, adoption of standard PSA models and data that do not addresses explicitly neither the effect of equipment ageing nor maintenance and testing programs could have a significant impact on the conclusions drawn from PSA studies and applications, particularly when NPP are operated at an advanced age or during long term operation. Thus, equipment ageing and asset management contributions would most likely result in large uncertainty of current component unreliability and unavailability models that support standard PSA quantification, particularly for aged equipment. Fortunately, these effects are limited often implicitly by adopting a living PSA or at least updating the standard PSA regularly, which is mandatory by current regulation in many countries.

It seems more appropriate that PSA modelling and data addresses above issues explicitly, instead of addressing them only implicitly at the most. Such issues can be seen evolving from the component level to the plant level. So, component reliability models must be developed first to support the Ageing PSA (APSA) modelling, which must integrate consideration of the combined effect of component ageing, maintenance management and technical specification requirements at NPP.

Pioneering work in this area started long time ago, which revealed the significance of equipment ageing what concerns PSA results and applications [7-11]. Nowadays, different approaches to Ageing PSA are being proposed to account for equipment ageing [12-14]. In this respect, lessons learnt on ageing assessment, modelling and management of SSC of NPP in the framework of international programs and initiatives (NPAR, GALL, IGALL, etc.) should be fully integrated when applicable within APSA models and data [7, 15, 16]. 
In this paper, a new approach to Ageing PSA modelling is proposed, which incorporates the impact of not only equipment ageing but also maintenance and testing policies explicitly into PSA models and data, which is intended to be used in the framework of the Risk Informed Decision Making philosophy (R.G. 1.174) [4]. This APSA could be used to support risk-informed decisions on the effectiveness of maintenance programs and technical specification requirements of critical equipment of Nuclear Power Plants (NPP), for example adopting the methodology proposed in Refs. [5, 6].

\section{EFFECTIVENESS OF MAINTENANCE AND TESTING}

Ref. [17] reviews briefly the role of maintenance and testing activities to achieve appropriate levels of reliability, availability and safety of safety related equipment at NPP. It illustrates basic concepts in relation to natural reliability, reliability degradation (e.g. ageing as degradation mechanism), inherent reliability, maintainability (e.g. maintenance types such as preventive, corrective and overhaul maintenance), availability, maintenance effectiveness (e.g. imperfect maintenance) and testing effectiveness (e.g. failures covered by surveillance tests). It pays special attention to describe both positive and negative effects of maintenance and testing.

\subsection{Effectiveness of maintenance activities}

Maintenance represents all activities performed on equipment in order to assess, maintain or restore its operational capabilities. Maintenance introduces two types of positive aspects. Firstly, corrective maintenance restores the operational capability of the failed or degraded equipment and secondly preventive maintenance increases the intrinsic reliability of non-failed equipment beyond the natural reliability, for example, controlling its degradation below the failure point. Although the equipment is subjected to preventive and corrective maintenance it may degrade over age depending on the working conditions of the equipment and the effectiveness of the maintenance being applied (so called imperfect maintenance). So that, several activities are scheduled to control evolution of degradation mechanisms that fall into the categories of continuous monitoring or periodic predictive maintenance, which are responsible for launching on condition preventive or corrective activities when necessary. 
On the contrary, maintenance also introduces adverse effects, called the downtime effect that represents the time the equipment is out of service to overcome maintenance (corrective, preventive, repair, overhaul, etc.). Thus, the adverse effect depends on the maintainability characteristics of the equipment. Maintainability represents the capability of the equipment or systems to be maintained under specified conditions during a given period, which depends to some extent on their physical characteristics or design among other factors, such as maintenance task force, spare parts, etc.

\subsection{Efficiency of surveillance requirements}

Surveillance requirements involves periodic tests, e.g. monthly or quarterly. A Test interval is established. The primary purpose of testing is to assure that equipment of safety systems normally in standby will be operable when needed in case of accident. By testing equipment, failures can be detected that may have occurred since the last test or the time when the equipment was last known to be operational. Then, positive effect of testing is its capability to detect hidden failures and this way limiting the risk of undetected downtimes of the safety component, i.e. the "test-limited" risk, which depends on not only the equipment unreliability characteristics, i.e. equipment failure rate, and the Surveillance Frequency (SF), i.e. test interval, but also on the effectiveness of testing, i.e. the capability of the test to cover only a fraction of hidden failures.

On the contrary, some tests may have an adverse impact on safety because of their undesirable effects, i.e. "test-caused" risk, such as, for example, test errors causing plant transients, wear out of equipment due to testing, etc. Often, a very important adverse effect is the one called the detected downtime effect that represents the time the equipment is out of service for testing. Thus, this adverse effect depends on the testing characteristics of the equipment. In general, the undesirable effects will be reduced if the SF is decreased, because then fewer tests will be conducted. By reducing the SF we also can obtain the additional benefit of reducing resources on testing. However, an important disadvantage here is that the fault-exposure time, i.e. the time during which the component will be subject to hidden failures during standby, will correspondingly increase as the SF decreases, i.e. the positive effect of the test limiting risk of undetected downtimes is reduced as well.

NPP safety systems consist of a number of redundant and diverse trains, each one consisting of highly reliable equipment, normally in standby, which must perform the intended safety function. Test Strategy 
establishes the grouping of equipment undertaken the test simultaneously, e.g. a full train, and the scheduling of the tests of the several groups, each group consisting of equipment in one of the redundant trains. Normally, the same SF applies to equipment of the redundant trains. However, the test-limited risk will depend on the relative scheduling of the tests of the redundant trains, i.e. the test strategy. Often, standard PSA quantification of the test-limited risk assumes that the relative test times of redundant components follow no specific schedule and are randomly placed with regard to one another. By staggering the test times of the components in different trains, then the test-limited risk will be reduced for the same SF as compared to the independent PSA assumption.

\subsection{NPP programs for improving effectiveness of maintenance and surveillance requirements}

Ref. [17] introduces ongoing programs and others being considered for implementation at NPPs. They can be grouped into two different groups, one that focuses on the improvement of licensing basis and the other that focuses on the improvement of maintenance activities. The first group includes Risk Informed Technical Specifications programs, which focuses on the improvement of Technical Specifications requirements such as Surveillance Testing Requirements and Completion Times. Other programs in this group focus on RiskInformed In-Service Inspection and Risk-Informed In-Service Testing following the same principles of the Risk Informed Decision Making philosophy proposed in RG 1.174. The second group of programs focuses on the improvement of the asset management and maintenance policy. A common objective of these programs is to reach and maintain a high intrinsic reliability target, reducing as much as possible the failure rate, which is of most relevance for SSCs important to NPP safety. This is one of the main purposes for implementing a Reliability Centered Maintenance program. Other programs in this group focus on Maintenance Rule Implementation, Life Management of SSC and License Renewal of NPP.

A common objective of all these programs is to allow reaching and keeping the most reliable, available and safe operation of NPP along it design life and beyond, if required, by means of implementing high effectiveness maintenance and testing activities. Effective maintenance programs and surveillance requirements are essential for ensuring acceptable equipment reliability and safe operation of ageing NPP along their service life; both design life and extended life for long-term operation. To ensure effectiveness of maintenance and testing programs, they should be periodically reviewed and modified based on operating experience and pref- 
erably making decisions based on their risk impact. Consequently, Ageing PSA modelling and data must be developed and updated taking into account explicitly the results of the implementation of such programs as they influence maintenance and testing effectiveness and this way they have a large influence on the evaluation of equipment reliability and NPP risk profiles over time $[8,9]$.

\section{RELIABILITY MODELLING ADDRESING EFFECTIVENESS OF MAINTENANCE AND TESTING}

\subsection{Failure rate model}

Traditionally, standard PSA of NPP assumes constant failure rate to modelling the reliability of components normally in standby

$\lambda(t)=\lambda_{0}$

In Ageing PSA, failure rate models must be formulated using age-dependent models, i.e. component reliability depends on its age, to account for the effect of component ageing. Different models of age-dependent reliability of components have been proposed in the literature [8-9, 18, 22-23].

The selection of the most adequate reliability model depends on aspects such as component type, its operational mode, the ageing mechanisms considered and maintenance and surveillance testing programs applied. As introduced in the previous section, effectiveness of maintenance programs and surveillance requirements are essential for ensuring acceptable component operational reliability and safe operation of nuclear power plants along their service life; both design life and extended life for long-term operation. Consequently, the reliability of the component should be modelled as a function of the inherent reliability of the component, i.e. component failure rate imposed by design, the component ageing, which degrades the inherent reliability, and the effectiveness of maintenance activities, which improve the reliability degraded by ageing, i.e. there is an attempt to return component reliability back to its inherent value eventually, but normally impossible, in case of perfect maintenance activities. In addition, the effectiveness of testing in relation to testing coverage of failure mechanism has to be taken into account also.

The following sections introduce a kind of age-dependent reliability model which address explicitly the effectiveness of maintenance and testing. 


\subsection{Age-dependent failure rate model addressing imperfect maintenance}

As proposed in $[18,19]$, it is supposed the age of a component, $w(t)$, evolves with chronological time, $t$. In addition, the effect of maintenance on the age of the component and on its reliability is included based on a model of imperfect maintenance. Imperfect maintenance models consider that each maintenance activity reduces the age of the component by some degree, depending on its effectiveness.

Among the different models of imperfect maintenance that can be found in the literature, this paper considers the Proportional Age Reduction (PAR) model and the Proportional Age Setback (PAS) model proposed in above Refs. $[18,19]$. The selection of the most appropriate model in each case depends on the component type, failure mechanism and sort of maintenance activity.

\subsubsection{Proportional Age Reduction based ageing and reliability models}

In the PAR approach, each maintenance activity is assumed to reduce proportionally the component age gained from the previous maintenance. In this model, the effect of maintenance is introduced by using an effectiveness parameter, $\varepsilon$, ranging in the interval $[0,1]$.

Assuming the time a component is out of service for maintenance is negligible as compared to the time between two consecutive maintenance activities, the evolution of the age of the component in the period $m+1$, between $m$ and $m+1$ maintenance activities, is given in general by [18]:

$w_{m+1}(t)=w_{m}^{+}+\left(t-t_{m}\right)$

where $w_{m}^{+}$is the age of the component immediately after maintenance number $m, t$ is the chronological time elapsed since the component was installed and $t_{m}$ is the time at which the m-maintenance activity is performed.

Under the PAR model, the age of the component in the period between maintenance activities $m$ and $m+1$ is given particularizing eqn. (2) to yield the following expression [18]:

$w_{m+1}(t)=t-\varepsilon \cdot t_{m}$

In the literature, one can find different component reliability models proposed to address the effect of equipment ageing, such as linear model, Exponential, Weibull, etc [8]. In this paper, the linear ageing failure rate model has been considered, being this the simplest age-dependent reliability model [19]. This model as- 
sumes that the failure rate has a linear behaviour with component age departing form an initial reliability, inherently by design, which can be expressed as $[9,19]$ :

$\lambda(w(t))=\lambda_{0}+\alpha \cdot w(t)$

where $t$ represents the chronological time, $\alpha$ is the linear ageing factor and $w(t)$ is the component's age given by eqn. (3) after maintenance $m+1$, which depends on the effectiveness of the maintenance policy applied on the component. In eqn. (4) component inherent failure rate by design is given by the term $\lambda 0$, which represents random failures, while the second term $\alpha \cdot w(t)$ represents the degradation of the equipment failure rate due to equipment ageing, which is counterbalanced by the effectiveness of the maintenance policy.

Substituting eqn. (3) into eqn. (4), the component age-dependent failure rate model is obtained under the PAR imperfect maintenance model after $m$-maintenance as follow:

$\lambda_{m+1}(t)=\lambda\left(w_{m+1}(t)\right)=\lambda_{0}+\alpha \cdot\left(t-\varepsilon \cdot t_{m}\right)$

Then, an average ageing failure rate, $\lambda^{*}{ }_{m+1}$, over the period between two consecutive maintenance activities, $m$ and $m+1$, can be obtained using the following expression:

$\lambda_{m+1}^{*}=\frac{1}{t_{m+1}-t_{m}} \int_{t_{m}}^{t_{m+1}} \lambda_{m+1}(t) d t$

which yields to

$\lambda_{m+1}^{*}=\lambda_{0}+\alpha \cdot \frac{M}{2}+\alpha \cdot(1-\varepsilon) \cdot t_{m}$

Eqn. (7) represents an averaged ageing failure rate between two consecutive maintenance activities, $m$ and $m+1$, which includes the effect of ageing using a linear ageing model $(\alpha)$ and the effect of the maintenance performed on the component modelled as a PAR model with constant maintenance effectiveness $(\varepsilon)$. In addition, $M$ represents a constant periodicity between maintenance activities, normally time-directed preventive maintenance.

\subsubsection{Proportional Age Setback based ageing and reliability models}

In the PAS approach, each maintenance activity is assumed to shift the origin of time from which the age of the component is evaluated. Thus, the PAS model considers that the maintenance activity reduces proportion- 
ally, in a factor of $\varepsilon$, the age that the component has immediately before it enters maintenance, where the maintenance effectiveness parameter $\varepsilon$ ranges in $[0,1]$.

Again, assuming the time a component is out of service for maintenance is negligible as compared to the time between two consecutive maintenance activities, the evolution of the age of the component in the period $m+1$, between $m$ and $m+1$ maintenance activities, is given by the following equation under the PAS model [17]:

$w_{m+1}(t)=t-\Delta w_{m}$

where

$\Delta w_{m}=\varepsilon \cdot \sum_{k=0}^{m-1}(1-\varepsilon)^{k} \cdot t_{m-k}$

Again, the linear ageing failure rate model has been considered. Then, substituting eqn. (8) into eqn. (4), the component ageing failure rate model is obtained under the PAS imperfect maintenance model after $m$ maintenance as follow:

$\lambda_{m+1}(t)=\lambda\left(w_{m+1}(t)\right)=\lambda_{0}+\alpha \cdot\left(t-\Delta w_{m}\right)$

Then, an average ageing failure rate, $\lambda^{*}{ }_{m+1}$, over the period between two consecutive maintenance activities, $m$ and $m+1$, can be obtained using eqns. (6) and (10) to yield:

$\lambda_{m+1}^{*}=\lambda_{0}+\alpha \cdot \frac{M}{2}+\alpha \cdot\left(t_{m}-\Delta w_{m}\right)$

Eqn. (11) represents an average ageing failure rate between two consecutive maintenance activities, $m$ and $m+1$, which includes the effect of the ageing using a linear ageing model $(\alpha)$ and the effect of the maintenance performed on the component modelled as a PAS model with constant maintenance effectiveness $(\varepsilon)$. In addition, $\mathrm{M}$ represents a constant periodicity between preventive maintenance activities and $\Delta \mathrm{Wm}$ is given by eqn. (9).

Based on the results found in [18], there is an asymptotic behaviour of the PAS model, then, eqn. (11) has an asymptotic behaviour given by:

$\lambda_{a}^{*}=\lambda_{0}+\alpha \cdot \frac{M}{2}\left(\frac{2-\varepsilon}{\varepsilon}\right)$ 


\subsection{Age-dependent failure rate model incorporating effectiveness of maintenance types}

As introduced in section 3, one can find several types of maintenance activities with an aim to assess, improve or restore component operational capability. Consequently, the maintenance effectiveness depends on the particular maintenance type being considered in each case.

In a corrective maintenance (CM) activity only the operational capability of the failed component failed is restored. Thus, it is often assumed that the age of the component after CM is exactly the same than that immediately before entering $\mathrm{CM}$, which means that the model of imperfect maintenance introduced in the previous section reduces to an "as Bad As Old (BAO)" model where the effectiveness of CM is assumed to be $\varepsilon=$ 0 .

On the opposite case, in an overhaul maintenance (OM) the component is replaced by a new one. Thus, it is often assumed that the age of the component after OM resets to zero, which means that the model of imperfect maintenance introduced in the previous section reduces to an "as Good As New (GAN)" model where the effectiveness of $\mathrm{OM}$ is assumed to be $\varepsilon=1$.

Last, preventive maintenance (PM) increases the intrinsic reliability of a non-failed component beyond the natural reliability, for example, controlling its degradation below the failure point. Thus, it is often assumed that the age of the component after PM is reduced as compared to that immediately before entering PM but without falling to zero. This means the model of imperfect maintenance introduced in the previous sections applies somewhere between BAO and GAN models, where $\varepsilon$ ranges in interval $] 0,1[$.

\subsection{Age-dependent failure rate model addressing test efficiency}

By testing, component failures can be detected that may have occurred since the last test or the time when the component was last known to be operational. The main objective of surveillance test is to detect hidden failures so that the component can be restored to its operational state, normally BAO state by performing CM after the test has detected a failure. A surveillance test is modelled here as allowing to assure to some extent; which depends on the test efficiency, that the component is operational, but without changing its age. Consequently, in reliability terminology, the surveillance test intervals are called BAO intervals since the component age coming out of the test is basically the same as the component age going into the test, i.e. the component is as old with regard to its age [8]. 
As introduced in the previous paragraph, test efficiency has to be taken into account. For example, as proposed in Ref. [20], it can be seen like a testing coverage of failure mechanisms, where coverage is defined as a share of detected an undetected failures by testing. Thus, test efficiency can be formulated in terms of the percentage of the total failure rate that is detected, i.e. covered, by testing, called also testing coverage. Alternatively, Ref. [9] defines test efficiency like the probability that a given failure is detected by the test.

In both previous cases, test efficiency can be represented by a single parameter $\eta$. Ref. [9] and [21] give values of $\eta$ for test efficiency of several component types. As a result, the consideration of a test efficiency splits the total age-dependent failure rate into two age-dependent failure rate modes: detected and undetected.

$$
\lambda_{m+1}^{*}=\eta \cdot \lambda_{m+1}^{*}+(1-\eta) \cdot \lambda_{m+1}^{*}
$$

where the test efficiency, $\eta$, ranges in the interval $[0,1]$. In eqn. (13), the first contribution represents the agedependent failure rate associated with detected failures by testing, $\lambda_{m+1}^{D}$, and the second part represents the age-dependent failure rate associated with undetected failures by testing, $\lambda_{m+1}^{U}$, which can be derived using the corresponding formulation for $\lambda_{m+1}^{*}$ as introduced in section 4.2 .

$$
\begin{aligned}
& \lambda_{m+1}^{D}=\eta \cdot \lambda_{m+1}^{*} \\
& \lambda_{m+1}^{U}=(1-\eta) \cdot \lambda_{m+1}^{*}
\end{aligned}
$$

On the other hand, a large number of critical components overtakes functional tests mostly performed during refuelling of NPP, where the refuelling Interval (RI) ranges between 12 and 24 months, so that typically RI could be set equal to 18 months. The functional test often involves testing full performance of the component capacity, so that it performs very close to real conditions in case of emergency. Then, the efficiency of such a functional test should be very close to one in detecting hidden failures. Similarly to the surveillance tests, in reliability terminology, the functional test intervals (often adopting the RI) are called BAO intervals since the component age coming out of the test is basically the same as the component age going into the functional test, i.e. the component is as old with regard to its age.

Thus, critical components for NPP safety may overtake at least two tests: one surveillance test and another functional test. Consequently, to address such a second or refuelling functional test, the undetected age- 
dependent failure rate, given by eqn. (15), should split into two new contributions: detected and undetected after the refuelling functional test, to yield

$$
\begin{aligned}
& \lambda_{m+1}^{U D}=\eta_{R I} \cdot \lambda_{m+1}^{U} \\
& \lambda_{m+1}^{U U}=\left(1-\eta_{R I}\right) \cdot \lambda_{m+1}^{U}
\end{aligned}
$$

where the test efficiency of the refuelling functional test, $\eta_{R}$, ranges also in the interval $[0,1]$, but very close to one now. In addition, eqn. (16) represents the age-depended failure rate contribution associated with detected failures only after the refuelling functional test, while eqn. (17) represents the age-dependent failure rate contribution associated with failures that remain undetected even after the refuelling functional test.

The formulation proposed in eqns. (13) to (17) can accommodate a number of assumptions made in standard PSA. For example, sometimes only the functional test is considered in standard PSA, which represents a conservative assumption where it is assumed implicitly that $\eta$ is set equal to 0 while $\eta_{R I}$ is set equal to 1 . Alternatively, when only surveillance testing is considered it is assumed implicitly that $\eta_{R I}$ is set equal to 0 . Care should be taken in this situation since in such a case it is often assumed at the same time that $\eta$ is set equal to 1, which is not a conservative assumption always. Therefore, as a general case, both contributions should be addressed as proposed in the following section.

\section{UNAVAILABILITY MODELLING}

As introduced above, Surveillance Requirement involves periodic tests, e.g. monthly or quarterly, with positive and negative effects [5]. The positive effect of testing is its capability to detect hidden failures and this way limiting the risk of undetected downtimes of the safety component, i.e. the "test-limited" risk, which depends on the equipment reliability, i.e. equipment failure rate, test efficiency, etc (see section 4.4) . Often, a very important adverse effect of testing, to be considered at the minimum, is the one called the detected downtime effect that represents the time the equipment is out of service for testing, i.e. "test-caused" risk. Thus, this adverse effect depends on the testing characteristics of the equipment. Note this adverse effect only applies for surveillance tests, while it does not apply for functional tests performed during refuelling of the NPP. 
On the other hand, in order to consider thoroughly the risk impact of maintenance, there is a need to consider not only its impact on component reliability (see sections 4.2 and 4.3) but also those component unavailability contributions related with equipment downtimes for maintenance, i.e. preventive maintenance, corrective maintenance, overhaul, etc., provided that they are not performed during refuelling of the NPP.

In summary, the unavailability contributions of a component normally in stand-by are divided into two categories: a) unavailability due to failures, i.e. unreliability effect, and b) unavailability due to testing and maintenance downtimes, named the downtime effect.

\subsection{Unreliability contributions}

Adopting the basis of the formulation of unreliability contributions in Refs. [18, 19] but addressing now the several age-dependent failure rate contributions introduced in section 4.4, the component unavailability due to unreliability contributions can be evaluated using the following equations :

$u_{m+1}^{D} \approx \frac{1}{2} \lambda_{m+1}^{D} \cdot T I$

$u_{m+1}^{U D} \approx \frac{1}{2} \lambda_{m+1}^{U D} \cdot R I$

$u_{m+1}^{U U} \approx \rho+\frac{1}{2} \lambda_{m+1}^{U U} \cdot L$

where $m+1$ represents the unavailability contributions are evaluated between maintenance activities $m$ and $m+1, u_{m+1}^{D}$ is the unreliability contribution due to detected failures by surveillance testing, $u^{U D_{m+1}}$ is the unreliability contribution due to undetected failures by surveillance testing that are then detected by a second functional tests and $u^{U U_{m+1}}$ is the unreliability contribution due to undetected failures by both surveillance and functional tests. In addition, the following notation has been used:

$\rho=$ cyclic or per-demand failure probability,

$\lambda^{D}{ }_{m+1}=$ detected fraction of age-dependent stand-by failure rate (section 4.4),

$\lambda^{U D_{m+1}}=$ undetected fraction of age-dependent stand-by failure rate that is then detected (section 4.4),

$\lambda^{U U_{m+l}}=$ undetected fraction of age-dependent stand-by failure rate that remains undetected (section 4.4),

$T I=$ surveillance test interval, 
$R I=$ functional test interval (considered herein equal to RI, refuelling interval)

$L=$ life of the component.

What concerns parameter $L$, it represents the component life as compared to the NPP design life. In case the component is not replaced by a new one over the NPP design life, then this parameter should be equal to the NPP design life. In case the component undertakes only time-directed overhaul maintenance, this parameter $L$ should be equal to a constant replacement period. Last, in case of the component being replaced based on its condition, e.g. failure rate going beyond a given threshold limit, this parameter $L$ should be equal to the component residual life.

\subsection{Downtime contributions}

Based on Refs. $[18,19]$ but addressing now only the age-dependent failure rate contribution corresponding to detected and repaired failures with the plant at power, as introduced in section 4.4 , the component unavailability due to detected downtimes for testing and maintenance contributions can be evaluated using the following equations:

$u_{m+1}^{T} \approx \frac{\tau}{T I}$

$u_{m+1}^{M} \approx \frac{\sigma}{M}$

$u_{m+1}^{C} \approx \lambda_{m+1}^{D} \cdot \mu$

$u_{m+1}^{O}=\frac{\Gamma}{L}$

where $m+1$ represents the unavailability contributions are evaluated between maintenance activities $m$ and $m+1, u_{m+1}^{T}$ represents the unavailability contribution due to testing, $u^{M+1}$ is the unavailability contribution due to performing preventive maintenance, $u_{m+1}^{C}$ is the unavailability contribution due to performing corrective maintenance, and $u_{m+1}^{O}$ is the contribution due to replacement of the equipment, if any. In addition, the following new notation has been used:

$\tau=$ downtime for testing, 
$\sigma=$ downtime for preventive maintenance,

$M=$ the period to perform time-directed preventive maintenance,

$\mu=$ downtime for repair when there are no time limitations on conducting such a repair,

$\Gamma=$ downtime for replacement, and

\section{RISK MODELLING}

There are different approaches that can be used for transforming a standard PSA to an age dependent PSA, or an APSA. Ref. [9] introduces three basic approaches. Herein, only two are introduced.

The first approach involves carrying out a standard PSA evaluation a number of times with different component failure rates used in each evaluation. The different component failure rates which are used are stepwise approximations to time dependent component failure rates. This approach is straightforward, but the age of each component cannot be separately tracked. Hence, the effect of testing, maintenance, or repair on the age of the component or on the ageing rate cannot be explicitly modelled. This is the approach adopted when using a Living PSA.

The second approach for transforming a PSA into an APSA is to substitute age dependent component reliability and unavailability models into the PSA quantification formulas. The fundamental PSA formulas for the Core Damage Frequency, CDF, in terms of the component failure probabilities and component unavailabilities are still used. However, the quantification formulas for the component failure probabilities and component unavailabilities are changed from the usual, steady state formulas, to those which explicitly incorporate ageing as is described in sections 4 and 5. This approach efficiently calculates the CDF and system unavailabilities as a function of plant age addressing explicitly the effects of maintenance and testing. This is the approach adopted in this paper.

Using a level 1 PSA, the corresponding risk metric for the evaluation of ageing is the annual average baseline CDF considering the effect component ageing, which can be re-formulated for a single component $i$ using the law of the total probability as follows:

$C D F=C D F_{i}^{0}+u_{i} \cdot\left(C D F_{i}^{1}-C D F_{i}^{0}\right)=C D F_{i}^{0}+u_{i} \cdot B_{i}$ 
where $u_{i}[-]$ represents the average unavailability of a given component $i, C D F_{i}^{l}\left[y e a r^{-1}\right]$ represents the increased risk with the component $i$ down, and $C D F_{i}^{0}\left[\right.$ year $\left.^{-1}\right]$ represents the reduced risk when the component $i$ is known not to be down, as compared both with the baseline risk, $C D F$ [year ${ }^{-1}$. Thus, age-dependent unavailability $u_{i}$ can be formulated by adding eqns. (18) to (24). In addition, $B_{i}$ corresponds to the traditional Birnbaum importance measure of component $i$.

On the other hand, RG 1.174 establishes two risk metrics for evaluating the risk impact of whatever change to the licensing basis [5], which are herein adapted to address the risk impact of equipment ageing in the example of application. Using a level 1 PSA, the required risk metrics for the evaluation of risk impact of ageing would be the annual average baseline CDF before ageing (named herein $C D F_{b}$ ) and the change in the annual average baseline CDF due to equipment ageing, which can be formulated as follows:

$$
\triangle C D F=C D F_{e}-C D F_{b}
$$

where $C D F_{b}$ and $C D F_{e}$ are the $\mathrm{CDF}$ at the beginning (b) and at the end (e) of a given period where the ageing effect is considered, respectively. Eqn. (26) can be particularized for the case of the single component $i$ by substituting eqn. (25) into eqn. (26) that yields:

$$
\triangle C D F_{i} \approx \Delta u_{i} \cdot B_{i}
$$

where

$$
\Delta u_{i}=u_{i}^{e}-u_{i}^{b}
$$

where $u_{i}^{b}$ and $u_{i}{ }^{e}$ are the average unavailability $u_{i}$ of the component $i$ at the beginning (b) and at the end (e) of a given period considering the ageing effect, respectively. Thus, $u_{i}^{b}$ and $u_{i}^{e}$ can be formulated by adding eqns. (18) to (24).

It should be noted that the age-dependent core damage frequencies, $C D F_{b}$ and $C D F_{e}$, are obtained using the level 1 APSA. $C D F_{e}$ will increase significantly due to cumulative ageing of several relevant components [8]. Therefore, the age-related unavailability contributions should be addressed at least for relevant components in the resulting Ageing-PSA.

As proposed in [12], the results of importance analysis performed for system components of the NPP, based on Fussell-Vesely importance measure, can be used to select the most critical components for ageing. Thus, it 
allows showing how the importance profile of components may drastically change due to ageing. Using eqn. (25), the Fussell-Vesely measure for component $i$ can be formulated as follows:

$$
F V_{i}=\frac{C D F-C D F_{i}^{0}}{C D F} \approx \frac{u_{i} \cdot B_{i}}{C D F}
$$

Eqn (29) shows the importance of component $i$ for the CDF depends on its unavailability, ui, and it functional/structural importance, $\mathrm{B}_{\mathrm{i}}$. Since $u_{i}$ changes with component's ageing, then its importance changes over NPP age too. Therefore, the importance profile of components will change according to the different ageing rates of the several components in a NPP. Fussell-Vesely importance measure can help in identifying the most critical components from those more affected by ageing, formulated as follows:

$$
F V_{i}^{\Delta C D F} \approx \frac{\Delta C D F_{i}}{\Delta C D F} \approx \frac{\Delta u_{i} \cdot B_{i}}{\Delta C D F}
$$

Despite of the interest of including the $n$ most critical components for NPP ageing into the formulation of the CDF, the scope of the example of application has been limited to better focus only on a single critical component as it is the simplest way to demonstrate the importance of addressing the effectiveness of maintenance and testing to evaluate the risk impact of equipment ageing.

\section{APPLICATION CASE}

\subsection{Problem description}

This section presents a simple example of application that focusses on analysing the risk impact of ageing of a single critical component of a NPP depending on several maintenance and surveillance alternatives along the last ten years of a NPP design life.

A motor-operated valve (MOV) of the Auxiliary Feed Water System (AFWS) has been selected based on several arguments. This valve is normally open and its function is to control the flow from AFWS until Steam Generators on the secondary of a typical Pressurized Water Reactor (PWR) NPP. First, the basic event representing MOV "fails to remain open" is one of the most important contributors to the CDF based on FussellVesely importance measure and using the standard PSA available. This basic event is modelled as a standby- 
related failure. Second, aging, maintenance, surveillance testing and functional test are meaningful for this MOV.

\subsection{Risk modelling and data}

A level 1 standard PSA at power considering only internal events is available, which allows obtaining CDF risk metric. The level 1 PSA used has been developed at component level, so that, the level of detail is appropriate to support the analysis of component ageing addressing effectiveness of maintenance and testing. However, there has been a need of refining the level 1 PSA modelling to address equipment ageing, i.e. to obtain the APSA, by replacing the standard failure rates and equipment unreliability and unavailability models by the new ones proposed in sections 4 and 5.

For sake of simplicity in the problem formulation and discussion of results, only the MOV of the AFWS is considered in order to formulate the APSA for this simple example of application. Table 1 shows data used for modelling the MOV basic event into the level 1 APSA for each Case considered within the sensitivity study performed. This table includes the description of parameters and corresponding constant value or probability distribution function (pdf). In the same way as in the standard PSA, all variables except of motoroperated failure rate have been considered constant, i.e. mean values have been used. MOV failure rate has been modelled as a random variable assuming a Gamma probability distribution function.

In Table 1 , the value of the linear ageing factor $(\alpha)$ has been obtained from a generic database, which is known as TIRGALEX [21]. TIRGALEX considers that the value of $\alpha$ in the case of motor-operated valves is equal to $4.11 \cdot 10^{-11} \mathrm{~h}^{-2}$. This value remains the same for Cases 1 to 7 . 
Table 1. Models and data used for representing the MOV basic event into the level 1 APSA for each Case 
In Table 1, one can find two groups of sensitivity cases. The first one consists of evaluating and comparing the risk impact of different Ageing-PSA models used actually in the context of incorporating ageing (without or with consideration of maintenance and test effectiveness) into standard PSAs (Case 1 to 3 ). Second one considers some sensitivity analysis cases to observe the effect of different testing and maintenance strategies on risk level, i.e. changing intervals and effectiveness (Case 4 to 7).

The first data are given for the original case, which considers the "current standard PSA", in which constant failure rate is used. Usually this value is updated on a regular basis at NPP to reflect the current design and operational features of the NPP over its design life, which is commonly known as Living-PSA. Therefore, adoption of standard PSA models and data does not addresses explicitly neither the effect that equipment ageing nor maintenance and testing effectiveness could have on the conclusions drawn from PSA studies and applications based on the analysis of the risk impact. As shown in Table 1, a test activity performed every plant shutdown with efficiency set to one is considered ( $T I=4616$ hours, which represents the mean time between plant shutdowns that is normally shorter than the refuelling period).

Case 1 takes into account the effect of component ageing, where asset management is addressed only implicitly into the ageing failure rate. Therefore, effectiveness of maintenance activities is not considered explicitly. Again, in this Case 1 only the test activity performed every plant shutdown with efficiency set to one is considered.

Case 2 extend the previous case to take into account also the effect of maintenance effectiveness explicitly. It is assumed a Proportional Age Reduction (PAR) model to represent the evolution of component age and reliability as a consequence of the time-directed preventive maintenance policy. A maintenance interval equal to 13140 hours has been stablished with maintenance effectiveness equal to 0,6. Again, the same test activity performed every plant shutdown with efficiency set to one is considered. This approach of ageing modelling is often used in the literature [22-23].

Simultaneous planning of both effective maintenance and efficient testing activities are necessary in order to allow timely mitigation and correction of detected active ageing effects and degradation as introduced in the previous sections. Case 3 has been formulated based on full development of models and parameters showed in sections 4 and 5 . As cited in the previous sections, test efficiency is an important parameter because it splits the total age-dependent failure rate into two age-dependent failure rate modes: detected and un- 
detected. The maintenance and testing data used in Case 3 has been obtained from literature data concerning equipment of similar characteristics in a typical Pressurized Water Reactor [8-9, 21]. Thus, in this case, two types of tests have been considered. The first one is a surveillance test with a Test interval (TI) and efficiency $(\eta)$, which consists of a service inspection for this valve being performed regularly every 3 months with the NPP at power. The second one is a functional test performed every refuelling period, which is represented by the refuelling Interval $(R I)$ and efficiency $\left(\eta_{R I}\right)$. Both tests are imposed by current Surveillance Requirements in the Technical Specifications for this MOV belonging to the AFWS. Moreover, as in the previous case timedirected preventive maintenance with periodicity $M$ equal to 13140 and corresponding effectiveness $\varepsilon$ equal to 0,6 have been considered.

Departing from this Case 3, a number of sensitivity analyses have been performed with an aim at demonstrating the risk impact of alternative maintenance and testing strategies (Case 4 to 7). Thus, Cases 4 and 5 focus on the effect of reducing the maintenance interval and improving the maintenance effectiveness, respectively. Cases 6 and 7 focus on the effect of reducing the test interval and improving its efficiency, respectively.

\subsection{Assessment of risk impact}

In this section, the CDF has been obtained using the standard PSA and using the APSA under different alternatives (Case 1 to Case 7) by adopting the corresponding data shown in Table 1 for each case. Thus, CDFb and $\triangle \mathrm{CDF}$ have been obtained using eqn. (25) and eqn. (26) as proposed in section 6 . Table 2 provides the results for each case. It shows $\mathrm{CDF}_{\mathrm{b}}$, which corresponds to the $\mathrm{CDF}$ at the beginning of the assessed period. In addition, Table 2 summarizes also the results for $\mathrm{CDF}_{\mathrm{e}}$, which correspond to the CDF at the end of the assessed period without or with consideration of ageing and effectiveness of testing and maintenance activities (Case 1 to 7). 


\subsection{Analysis of risk impact}

In this section, risk assessment results are compared against acceptance guidelines in RG 1.174. Couples of values $\left\{\mathrm{CDF}_{\mathrm{b}}, \triangle \mathrm{CDF}\right\}$ are used to evaluate the risk impact of equipment ageing for each Case.

According to RG 1.174, the appropriate numerical measures to use in the comparison of the PSA results to the acceptance guidelines are mean values. Those mean values refer to the means of the probability distributions that result from the propagation of the uncertainties on the input parameters and those model uncertainties explicitly represented in the model (middle point for each couple in Figures 1 and 2).

The analysis of the results in this section has been divided into two parts. The first one is devoted to compare the results of the evaluation of risk impact of component ageing using the standard PSA and the different approaches for Ageing-PSA discussed in this work (Case 1 to 3). Figure 1 presents the results of such comparison.

In Figure 1, one can observe couples $\left\{\mathrm{CDF}_{b}, \Delta \mathrm{CDF}\right\}$ for each case, Standard PSA and Ageing-PSA (Case 1 to 3). As expected, Standard PSA cannot capture the effect of component ageing, so it remains at the bottom line where $\triangle \mathrm{CDF}$ equals to zero. On the contrary, Case 1 to 3 are located in Region II according to RG 1.174. Case 3 is the reference case in this study as it adopts the Ageing-PSA approach proposed in this paper, which addresses explicitly and simultaneously the impact of component ageing, maintenance effectiveness and test efficiency. Case 1 address component ageing but it does not address neither maintenance effectiveness nor test efficiency and consequently it overestimate the risk impact of component ageing, $\triangle \mathrm{CDF}$. On the contrary, Case 2 considers both equipment ageing and maintenance effectiveness explicitly, but it does not account for test efficiency, so that it underestimate the risk impact of component ageing, $\Delta \mathrm{CDF}$.

Figure 1: Evaluation on risk impact of ageing depending on the APSA model used (A=Ageing, $\mathrm{ME}=$ Maintenance Effectiveness, $\mathrm{TE}=$ Test Efficiency)

As introduced above, Case 1 to 3 are located in Region II according to RG 1.174 (see Figure 1). In this region, as established in this Regulatory Guide, improvements should be given in order to move the couple 
from this region to Region III (acceptable). Therefore, it may seem appropriate to implement some kind of compensatory measure for ageing management, for example, adopting improved maintenance and testing strategies.

This way, the second part of this study adopts the Ageing-PSA approach proposed in this paper (Case 3). It presents the results of sensitivity analysis cases developed using this approach in order to show it is possible to assess explicitly the risk impact of component ageing depending on alternative testing and the maintenance strategies (Case 4 to 7), which could be used in the framework of risk informed decision making (RIDM) on changes to testing and maintenance policies.

A key aspect of NPP life management in a RIDM context is to evaluate the risk impact of existing maintenance and testing programs in managing ageing of systems, structures and components. Eventually, if compensatory measures are necessary to managing ageing in an appropriate way, it should include also assessment of the effectiveness of managing equipment ageing by adopting alternative maintenance management programs and/or reformulation of current operational requirements such as surveillance requirements. In this context, four sensitivity analysis cases are presented in this work (Case 4 to 7 ), each focused on improving maintenance or testing strategies. The results are shown in Figure 2.

Figure 2. Sensitivity analysis to Case \# $\{\mathrm{M}, \varepsilon, \mathrm{TI}, \eta\}$

Case 4 and Case 5 are focused on analysing the risk impact of component ageing departing from the reference case (Case 3) and introducing some changes in current maintenance policy, i.e. by reducing maintenance interval (Case 4) and by increasing maintenance effectiveness (Case 5), respectively. In Case 4, the maintenance interval, M, is reduced from 13140 hours to 4320 hours while other parameters keep at the same values as for Case 3. One can observe in Figure 2 the risk impact of component ageing, $\triangle \mathrm{CDF}$, for this case is reduced. In Case 5, the maintenance effectiveness is increased as compared to Case 3 while other parameters keep constant at the same values. One can observe in Figure 2 the risk impact of component ageing decreases significantly moving the couple $\left\{\mathrm{CDF}_{\mathrm{b}}, \Delta \mathrm{CDF}\right\}$ to acceptable region (Region III).

Current Technical Specifications impose surveillance testing of this MOV every 2160 hours (approximately three months). Case 6 and Case 7 focus on analysing the risk impact of component ageing departing from 
the reference case (Case 3) and introducing some changes in current testing policy, i.e. by reducing test interval (Case 6) and by increasing test efficiency (Case 7), respectively. In Case 6, the test interval is reduced from 2160 hours to 720 hours, while the functional test and other parameters remains the same values as for the reference case (Case 3). In Figure 2, one can observe the risk impact of component ageing, $\Delta \mathrm{CDF}$, for this case is reduced slightly. In Case 7, test efficiency is increased from 0,6 to 0,9. As one can observed in the Figure 2, the reduction of $\triangle \mathrm{CDF}$ is more significant now as compared with the one observed for Case 6 moving the couple $\left\{\mathrm{CDF}_{\mathrm{b}}, \Delta \mathrm{CDF}\right\}$ to the acceptable region (Region III).

\section{CONCLUDING REMARKS}

This paper presents a new approach to Ageing PSA (APSA) modelling to be considered in the framework of the Risk Informed Decision Making according to R.G. 1.174, which is intended to be used to support riskinformed decisions on the effectiveness of asset management programs and technical specification requirements of critical equipment of Nuclear Power Plants (NPP). This APSA can help in performing the Periodic Safety Review to support risk-informed decisions required to obtain approval from the regulatory body for continuing NPP operation. This APSA incorporates not only equipment ageing but also maintenance and surveillance testing requirements explicitly into APSA models and data as compared to standard PSA.

The CDF evaluation has been developed using age-dependent reliability, availability and risk models and data of a typical PWR, which allows obtaining results that are more realistic. The results are analysed in the framework of risk-informed decision-making established in RG 1.174. Thus, the results obtained from the different cases of application show how the risk impact of ageing depends on not only ageing of critical equipment but also on maintenance and testing practices. This way, the APSA allows addressing explicitly the role of compensatory measures to component ageing in terms of improved maintenance programs and changes on surveillance requirements, which allows satisfying safety criteria as imposed by current NPP regulation within this framework.

Finally, this paper presents a preliminary study in which only one critical component has been considered into the APSA. Future work should consider at least all critical components and use plant-specific ageing data in order to obtain plant-specific robust results, while allowing to consider the aggregate effect of ageing and maintenance and testing programs. Note sensitivity analyses have shown the risk impact depends largely on 
the model parameters, such as ageing factor, maintenance effectiveness, test efficiency and so one. Thus, data for support of ageing PSA is difficult to be obtained and significant uncertainty may be involved in the results as a consequence of the uncertainty behind estimation of ageing rate, maintenance effectiveness and test efficiency. Then, plant-specific data should be used to the extent possible in order to reduce uncertainty in quantifying the risk impact of component ageing.

\section{ACKNOWLEDGMENTS}

Authors are grateful to the Spanish Ministry of Science and Innovation for the financial support of this work (Research Project ENE2013-45540-R) and the doctoral fellow (BES-2011-043906).

\section{REFERENCES}

[1] The European Strategic Energy Technology Plan SET-Plan. Communication from the Comission to the European Parliament. [SWD(2013) 157]

[2] Strategic Research Area. Mandate for the Nuclear GEN II and III associations. November 2011

[3] Strategic Research and Innovation Agenda. NUGENIA. Sustainable Nuclear Energy Technology Platform (SNETP). February 2013.

[4] U.S. Nuclear Regulatory Commission. An Approach for Determining the Technical Adequacy of Probabilistic Risk Assessment Results for Risk-Informed Activities. RG 1.174. Revision 2. Washington DC, 2011.

[5] Martorell S., Villamizar M., Marton I., Sanchez A., Carlos S., Villanueva J.F. Evaluation of risk impact of changes to surveillance requirements addressing model and parameter uncertainties. Reliability Engineering and System Safety 2014; 126: 153-165.

[6] Martorell S., Marton I., Villamizar M., Sanchez A., Carlos S., Evaluation of risk impact of changes to completion times addressing model and parameter uncertainties. Reliability Engineering and System Safety 2014; 130: 190-201.

[7] U.S. Nuclear Regulatory Commission. Nuclear Plant Aging Research (NPAR) Program Plan, NUREG1144, US NRC, Washington, D.C, 1991 
[8] Vesely W.E., Kurth R.E., Scalzo S.M. Evaluations of core melt frequency effects due to component ageing and maintenance. NUREG/CR-5510. Washington. DC: USNRC, 1990.

[9] U.S. Nuclear Regulatory Commission. Approaches for Age-Dependent Probabilistic Safety Assessments with Emphasis on Prioritization and Sensitivity Studies. NUREG/CR-5587. SAIC-92/1137. Washington DC, 1992.

[10] Kim IS., Martorell S., Vesely WE., Samanta PK. Quantitative evaluation of surveillance test intervals including test-caused risks. NUREG/ CR-5775. Washington: US NRC. 1992.

[11] Kim IS., Martorell S., Vesely WE., Samanta PK. Risk analysis of surveillance requirements including their adverse effects. Reliability Engineering and System Safety 1994; 45: 225-234.

[12] European Comission, JRC Science and Policy Reports, Feasibility Study for applicability of Ageing PSA model results in Risk-informed Decision Process, APSA Network, 2014

[13] Volkanovski A. Method for assessment of ageing based on PSA results, Nuclear Engineering and Design 2012; 246: 141-146.

[14] Cepin M., Volkanovski A., Consideration of ageing within probabilistic safety assessment models and results, Kerntechnik, 2009, vol. 74, no. 3, pp. 140149.

[15] U.S. Nuclear Regulatory Commission. Generic Aging Lessons Learned (GALL) Report, NUREG-1801 U.SNRC, 2001

[16] International Atomic Energy Agency (IAEA), 2013. International Generic Ageing Lessons Learned (IGALL) for Nuclear Power Plants, IAEA Safety Report, DD1085, Vienna, 2013.

[17] Martorell, S., Villanueva, J. F., Carlos, S., Nebot, Y., Sánchez, A., Pitarch J. L. and Serradell, V. RAMS $+\mathrm{C}$ informed decision-making with application to multi-objective optimization of technical specifications and maintenance using genetic algorithms. Reliability Engineering \& System Safety 2005; $87(1): 65-75$.

[18] Martorell S., Sanchez A., Serradell V. Age-dependent reliability model considering effects of maintenance and working conditions. Reliability Engineering and System Safety 1999; 64: 19-31.

[19] Sanchez A., Carlos S., Martorell S., Villanueva J.F. Addressing imperfect maintenance modelling uncertainty in unavailability and cost based optimization. Reliability Engineering and System Safety 2009; 94 (1): $22-32$. 
[20] A.C. Torres-Echeverría, S. Martorell, H.A. Thompson. Modelling and optimization of proof testing policies for safety instrumented systems. Reliability Engineering and System Safety 94 (2009) 838-854.

[21] Levy. I. et al S., Wreathall. J., DeMoss., G..Wolford. A. J. Collins. E. P., \& Jarrell. D. B. (1988). Prioritization of TIRGALEX recommended components for further ageing research. NUREG/CR-5248. Washington. DC: US NRC.

[22] Kancev D., Cepin M. (2011). Evaluation of risk and cost using an age-dependent unavailability modelling of test and maintenance for standby components. Journal of Loss Prevention in the Process Industries $2011,24\left(2^{\perp}\right) ; 146-155$

[23] Kancev D., Cepin M. (2012). Uncertainty and sensitivity analyses for age-dependent unavailability model integrating test and maintenance. Nuclear Engineering and Design 2012, 246; 158-135

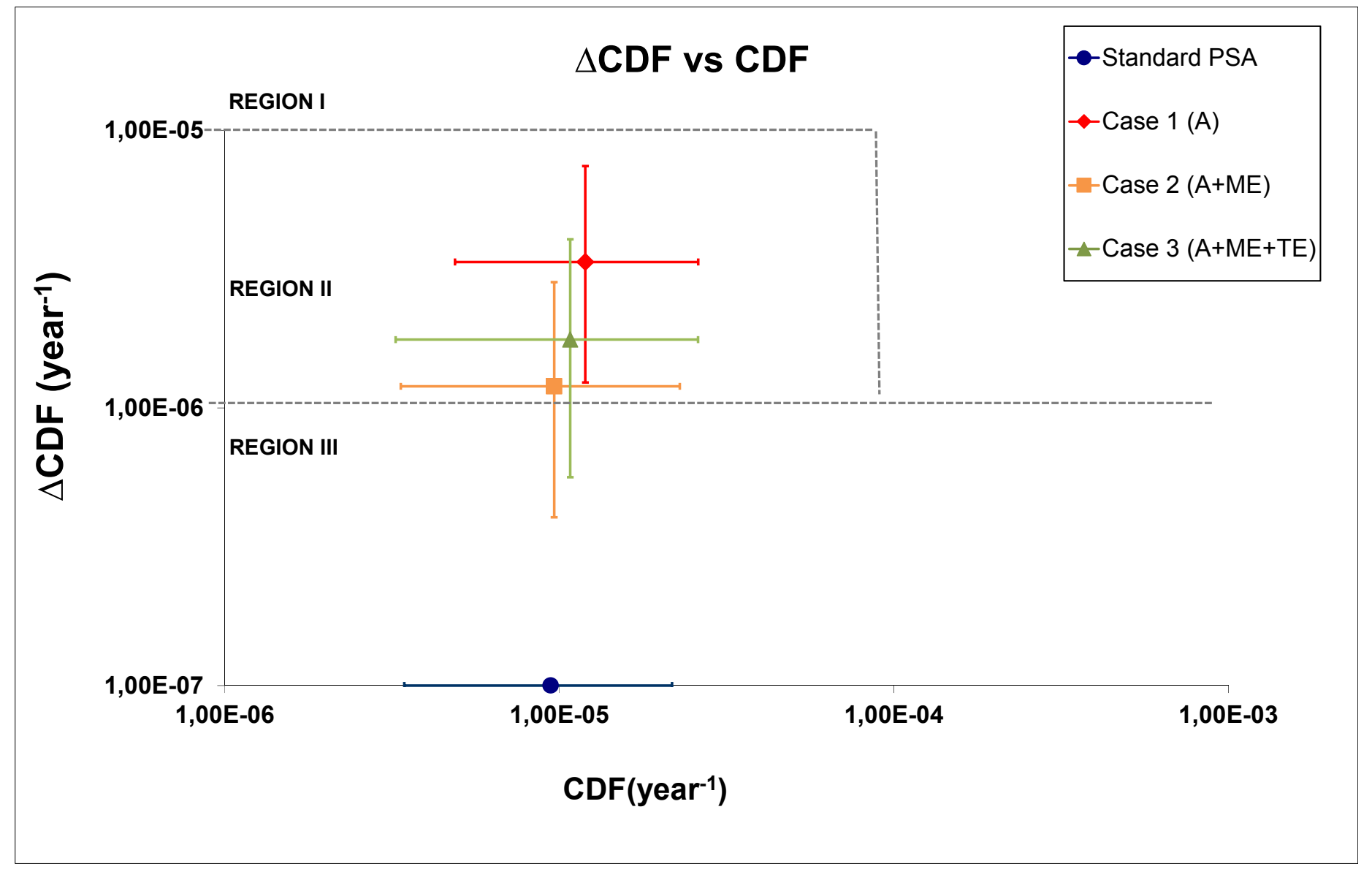

Figure 1: Evaluation on risk impact of ageing depending on the APSA model used (A=Ageing, $\mathrm{ME}=$ Maintenance Effectiveness, $\mathrm{TE}=$ Test Efficiency) 


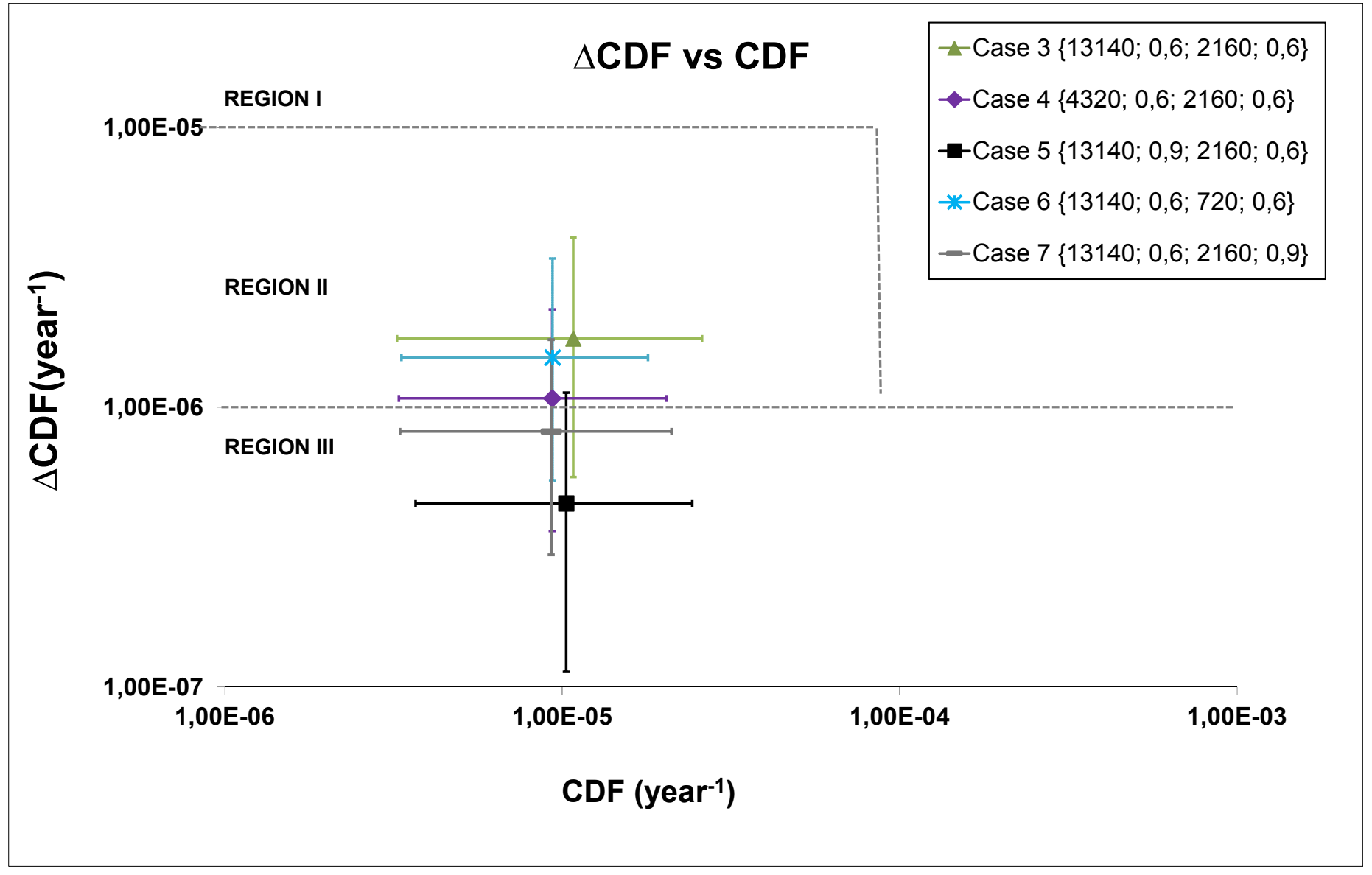

Figure 2. Sensitivity analysis to Case $\#\{M, \varepsilon, T I, \eta\}$ 
Table 1. Models and data used for representing the MOV basic event into the level 1 APSA for each Case

\begin{tabular}{|c|c|c|c|c|c|c|c|c|c|c|}
\hline Parameter & Description & $\begin{array}{c}\text { Parameter } \\
\text { type }\end{array}$ & Current PSA & Case 1 & Case 2 & Case 3 & Case 4 & Case 5 & Case 6 & Case 7 \\
\hline$\lambda_{0}\left(\mathrm{~h}^{-1}\right)$ & $\begin{array}{l}\text { Motor Oper- } \\
\text { ated Valves } \\
\text { initial failure } \\
\text { rate }\end{array}$ & pdf & $\begin{array}{c}\operatorname{Gamma}(4.45 \cdot \mathrm{E}- \\
08,0.5)\end{array}$ & $\begin{array}{c}\operatorname{Gamma}(4.45 \cdot \mathrm{E}- \\
08,0.5)\end{array}$ & $\begin{array}{c}\operatorname{Gamma}(4.45 \cdot \mathrm{E}- \\
08,0.5)\end{array}$ & $\begin{array}{c}\operatorname{Gamma}(4.45 \cdot \mathrm{E}- \\
08,0.5)\end{array}$ & $\begin{array}{c}\operatorname{Gamma}(4.45 \cdot E- \\
08,0.5)\end{array}$ & $\begin{array}{c}\operatorname{Gamma}(4.45 \cdot \mathrm{E}- \\
08,0.5)\end{array}$ & $\begin{array}{c}\operatorname{Gamma}(4.45 \cdot E- \\
08,0.5)\end{array}$ & $\begin{array}{c}\operatorname{Gamma}(4.45 \cdot E- \\
08,0.5)\end{array}$ \\
\hline$\alpha\left(h^{-2}\right)$ & $\begin{array}{l}\text { Ageing Fac- } \\
\text { tor }\end{array}$ & constant & No ageing & $4.11 \cdot E-10$ & $4.11 \cdot E-10$ & $4.11 \cdot E-10$ & $4.11 \cdot E-10$ & $4.11 \cdot E-10$ & $4.11 \cdot E-10$ & $4.11 \cdot E-10$ \\
\hline M (h) & $\begin{array}{l}\text { Maintenance } \\
\text { Interval }\end{array}$ & constant & No & No & 13140 & 13140 & 4320 & 13140 & 13140 & 13140 \\
\hline$\varepsilon$ & $\begin{array}{l}\text { Maintenance } \\
\text { effectiveness }\end{array}$ & constant & No & No & 0,6 & 0,6 & 0,6 & 0,9 & 0,6 & 0,6 \\
\hline TI (h) & Test Interval & constant & 4616 & 4616 & 4616 & 2160 & 2160 & 2160 & 720 & 2160 \\
\hline$\eta$ & TI efficiency & constant & 1 & 1 & 1 & 0,6 & 0,6 & 0,6 & 0,6 & 0,9 \\
\hline RI (h) & $\begin{array}{l}\text { Functional } \\
\text { test interval }\end{array}$ & constant & No & No & No & 13140 & 13140 & 13140 & 13140 & 13140 \\
\hline$\eta_{\mathrm{RI}}$ & $\begin{array}{l}\text { Test effi- } \\
\text { ciency of the } \\
\text { functional } \\
\text { test }\end{array}$ & constant & No & No & No & 1 & 1 & 1 & 1 & 1 \\
\hline
\end{tabular}


Table 2. Results of CFD and $\triangle \mathrm{CDF}$ for each Case (10 years period considered)

\begin{tabular}{|c|c|c|c|c|c|c|c|c|c|}
\hline & \multicolumn{3}{|c|}{$\mathrm{CDF}_{\mathrm{b}}\left(\right.$ year $\left.^{-1}\right)$} & \multicolumn{3}{|c|}{$\mathrm{CDF}_{\mathrm{e}}\left(\right.$ year $\left.^{-1}\right)$} & \multicolumn{3}{|c|}{$\mathrm{ACDF}=\mathrm{CDF}_{\mathrm{e}}-\mathrm{CDF}_{\mathrm{b}}(10$ years $)$} \\
\hline & Mean & $5 \%$ Perc. & $95 \%$ Perc. & Mean & $5 \%$ Perc. & $95 \%$ Perc. & Mean & $5 \%$ Perc. & $95 \%$ Perc. \\
\hline Standard PSA & $9,42 \mathrm{E}-06$ & $3,44 \mathrm{E}-06$ & $2,17 \mathrm{E}-05$ & $9,42 \mathrm{E}-06$ & $3,44 \mathrm{E}-06$ & $2,17 \mathrm{E}-05$ & - & - & - \\
\hline Case 1 & $1,20 \mathrm{E}-05$ & $4,88 \mathrm{E}-06$ & $2,60 \mathrm{E}-05$ & $1,64 \mathrm{E}-05$ & $6,35 \mathrm{E}-06$ & $3,65 \mathrm{E}-06$ & $3,35 \mathrm{E}-06$ & $1,24 \mathrm{E}-06$ & $7,44 \mathrm{E}-06$ \\
\hline Case 2 & $9,65 \mathrm{E}-06$ & $3,36 \mathrm{E}-06$ & $2,29 \mathrm{E}-05$ & $1,10 \mathrm{E}-05$ & $3,68 \mathrm{E}-06$ & $2,50 \mathrm{E}-05$ & $1,20 \mathrm{E}-06$ & 4,04E-07 & $2,84 \mathrm{E}-06$ \\
\hline Case 3 & $1,08 \mathrm{E}-05$ & $3,24 \mathrm{E}-06$ & $2,60 \mathrm{E}-05$ & $1,24 \mathrm{E}-05$ & $3,71 \mathrm{E}-06$ & $2,86 \mathrm{E}-05$ & $1,76 \mathrm{E}-06$ & $5,63 \mathrm{E}-07$ & $4,05 \mathrm{E}-06$ \\
\hline Case 4 & $9,34 \mathrm{E}-06$ & $3,28 \mathrm{E}-06$ & $2,04 \mathrm{E}-05$ & $1,04 \mathrm{E}-05$ & $3,69 \mathrm{E}-06$ & $2,25 \mathrm{E}-05$ & $1,08 \mathrm{E}-06$ & $3,62 \mathrm{E}-07$ & $2,24 \mathrm{E}-06$ \\
\hline Case 5 & $1,03 \mathrm{E}-05$ & $3,68 \mathrm{E}-06$ & $2,43 \mathrm{E}-05$ & $1,11 \mathrm{E}-05$ & $3,56 \mathrm{E}-06$ & $2,67 \mathrm{E}-05$ & $4,53 \mathrm{E}-07$ & $1,13 \mathrm{E}-07$ & $1,13 \mathrm{E}-06$ \\
\hline Case 6 & $9,36 \mathrm{E}-06$ & $3,34 \mathrm{E}-06$ & $1,80 \mathrm{E}-05$ & 1,19E-05 & $4,06 \mathrm{E}-06$ & $3,01 \mathrm{E}-05$ & $1,51 \mathrm{E}-06$ & $5,45 \mathrm{E}-07$ & $3,40 \mathrm{E}-06$ \\
\hline Case 7 & $9,28 \mathrm{E}-06$ & 3,31E-06 & $2,11 \mathrm{E}-05$ & $1,03 \mathrm{E}-05$ & 3,71E-06 & $2,46 \mathrm{E}-05$ & $8,20 \mathrm{E}-07$ & 2,98E-07 & $1,75 \mathrm{E}-06$ \\
\hline
\end{tabular}

